\title{
Improving selectivity of free electron maser with 1D Bragg resonator using coupling of propagating and trapped waves
}

\author{
N. S. Ginzburg, A. M. Malkin, N. Yu. Peskov, and A. S. Sergeev \\ Institute of Applied Physics RAS, 46 Uljanov Street, Nizhny Novgorod 603950, Russia
}

\author{
A. K. Kaminsky, S. N. Sedykh, E. A. Perelshtein, A. P. Sergeev, and A. V. Elzhov \\ Joint Institute for Nuclear Research, Dubna 141980, Russia \\ (Received 31 December 2004; published 28 April 2005)
}

\begin{abstract}
A novel scheme of the Bragg free-electron maser (free-electron laser) [FEM (FEL)] based on the coupling of an amplified propagating wave with a quasicutoff mode trapped inside the cavity is considered. The cutoff mode is essential for FEM self-excitation while energy extraction from the electron beam in the steady-state oscillation regime is almost completely determined by the propagating mode, synchronous to the beam. The main advantage of the discussed scheme over the traditional scheme of Bragg FEM is improving selectivity over the transverse index. Based on the proposed feedback scheme the advance of the JINR-IAP FEM to the shorter wavelength is discussed.
\end{abstract}

DOI: 10.1103/PhysRevSTAB.8.040705

PACS numbers: 41.60.Cr, 42.25.Fx

\section{INTRODUCTION}

A number of oscillator schemes based on the interaction between propagating and quasicutoff trapped waves are known in microwave electronics. One of them is a scheme of a gyrotron [1] where an electron beam excites a quasicutoff mode while the output of radiation is provided by the propagating wave coupled with the trapped one via corrugation of the waveguide sidewalls (in the case of helical corrugation, a similar scheme has been used recently for the band enhancement in gyroamplifiers [2]). One more example is a scheme of cyclotron resonance maser (CRM) [3] and free-electron laser (FEL) [4] in which the electron beam interacts both with a propagating wave (at the first harmonic) and a cutoff mode wave (at the second or third harmonic). In this case direct coupling of electromagnetic waves is absent and the waves interact via electron beam modulation. The trapped cutoff mode provides an oscillator self-excitation while the propagating wave is responsible for energy extraction in a steady-state regime.

In this paper we discuss a new variant of free-electron maser (FEM) based on the interaction between a propagating wave and a quasicutoff trapped wave [5]. In a suggested scheme a beam of wiggling electrons interacts only with a propagating wave, but the latter is coupled to a quasicutoff mode. This coupling could be realized by either helical or azimuthally symmetric periodical waveguide corrugation. The quasicutoff mode provides the feedback mechanism leading to the self-excitation of the whole system while the efficiency in the steady-state regime of generation is almost completely determined by the interaction with the propagating wave, synchronous to the beam.

The main advantage of the suggested scheme is the provision of a higher selectivity over the transverse index than a traditional scheme of FEM with Bragg resonators where a feedback wave propagates in a backward direction with rather high group velocity [6]. At the same time, this scheme is able to provide higher Doppler up-shift in comparison with the scheme discussed in $[3,4]$, where the frequency is restricted by a number of the operating harmonic. The novel feedback scheme will be tested at a JINR-IAP FEM $[7,8]$ as a method of increasing oscillation frequency for fixed transverse size of interaction space.

\section{BASIC MODEL}

Let us consider the simplest model of FEM oscillator exploiting the coupling of a propagating and a cutoff mode on periodical corrugation. Because of a small variation of the waveguide mean radius at the boundaries of the interaction space the cutoff mode is trapped inside the cavity as shown in Fig. 1. The field of this mode can be presented in the form

$$
\vec{E}=\operatorname{Re}\left[B(t) \vec{E}_{B}\left(r_{\perp}\right) f(z) e^{-i m_{B} \varphi} e^{i \omega_{0} t}\right] .
$$

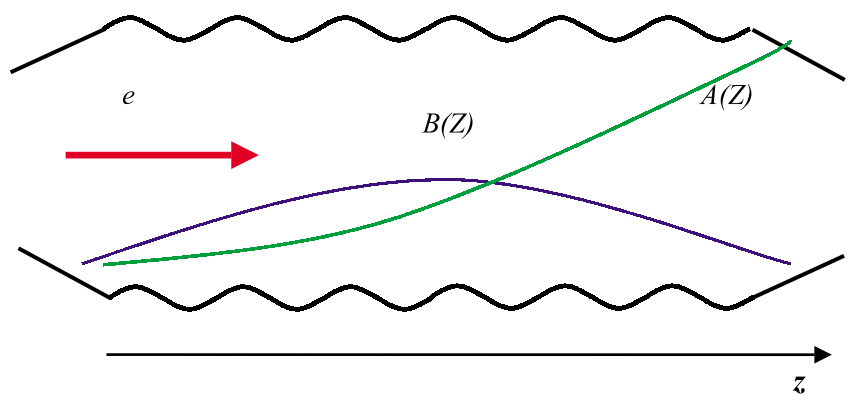

FIG. 1. (Color) Scheme of Bragg FEM based on the coupling of propagating (green line) and cutoff (blue line) modes on the cylindrical waveguide with sidewalls corrugations. Because of a small variation of the waveguide mean radius the cutoff mode is trapped inside the cavity. 
The forward propagating wave

$$
\vec{E}=\operatorname{Re}\left[A(t, z) \vec{E}_{\mathrm{A}}\left(r_{\perp}\right) e^{-i h_{0} z-i m_{A} \varphi} e^{i \omega_{0} t}\right]
$$

is synchronous to the electrons moving in the $+z$ direction. A corresponding synchronism condition can be presented as

$$
\omega_{0}-h_{0} v_{\|} \approx \Omega,
$$

where $\Omega$ is the frequency of particle oscillations in a spatially periodic undulator field for FEM (FEL) or in a homogenous magnetic field for the cyclotron resonance maser. In (1) and (2) $\omega_{0}$ is the carrier frequency, which is chosen equal to the eigenfrequency of the trapped mode, $\vec{E}_{A, B}\left(r_{\perp}\right)$ functions specify the transverse structure of the modes.

Helical corrugation

$$
r=a+a_{1} \cos (\bar{h} z+\bar{m} \varphi)
$$

( $a$ is a mean radius of the waveguide, $\bar{h}=2 \pi / d, d$ and $2 a_{1}$ are the period and the depth of the corrugation, correspondingly) under the Bragg resonance conditions

$$
\begin{aligned}
h_{0} & \approx \bar{h}, \\
\bar{m} & =m_{A}-m_{B}
\end{aligned}
$$

provides the coupling of the waves (1) and (2). A dispersion diagram, showing the coupling of partial waves is presented in Fig. 2. Taking into account that the eigenfrequency of the feedback mode (1) is close to the cutoff frequency $\omega_{0} \approx \omega_{c}=\left(c \nu_{B} / a\right)$, from (5a) we obtain a relation on the geometrical parameters of the system

$$
\frac{2 \pi}{d} \approx \frac{\sqrt{\nu_{B}^{2}-\nu_{A}^{2}}}{a},
$$

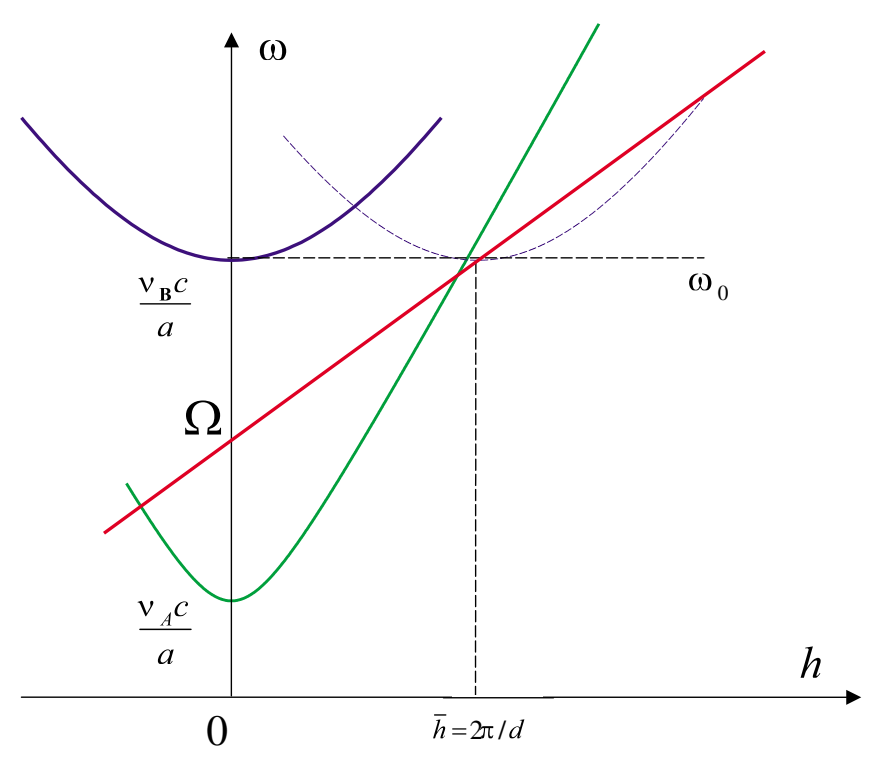

FIG. 2. (Color) Dispersion diagrams, showing the coupling of propagating (green curve) and cutoff (blue curve) partial waves. The red line is an electron wave $\omega=\Omega+h v_{\|}$. where $\nu_{\mathrm{A}, \mathrm{B}}$ are the roots of Bessel functions (or their derivatives) corresponding to the modes of a regular waveguide.

Assuming that the quality factor $Q$ of the trapped mode (1) is high, one can consider its longitudinal structure $f(z)$ fixed, unlike the longitudinal structure of the propagating mode $A(z)$, which is changed under the influence of the electron beam. In this case the process of amplification of synchronous wave and the mutual coupling of the propagating (2) and trapped (1) modes can be described by the following system of equations:

$$
\begin{gathered}
\frac{\partial \hat{A}}{\partial Z}=i \alpha \hat{B} f(Z) e^{i \delta Z}+J, \\
\frac{\partial \hat{B}}{\partial \tau}+\frac{1}{2 Q} \hat{B}=-i \alpha \int_{0}^{L} \hat{A}(Z) e^{-i \delta Z} f^{*}(Z) d Z .
\end{gathered}
$$

Here $J=\frac{1}{\pi} \int_{0}^{2 \pi} e^{-i \theta(Z)} d \theta_{0}$ is an amplitude of rf current, which is determined by the electron motion equations, where $\theta=\omega_{0} t-h_{0} z-\int \Omega d t$ is the electron phase in the field of the synchronous wave, and $\theta=\omega_{0} t_{0}$ is the initial phase. In the case of relatively small changes in electron energies the averaged motion equations can be presented in the form [6]

$$
\frac{\partial^{2} \theta}{\partial Z^{2}}=\operatorname{Re}\left[\hat{A}(Z) e^{i \theta}\right]
$$

In Eqs. (7) and (8) we have used the following dimensionless variables and parameters: $Z={ }_{z} C \bar{h}, \tau=\omega_{0} t, \hat{B}=$ $e \kappa \mu B \sqrt{N_{A}} / m c \gamma_{0} C^{2} \sqrt{\omega_{0} N_{B}}, \hat{A}=e \kappa \mu A / m c \omega_{0} \gamma_{0} C^{2}$.

$$
C=\left(\frac{e I_{0}}{m c^{3}} \frac{c \lambda^{2} K^{2} \mu}{4 \pi^{2} \gamma_{0} N_{A}}\right)^{1 / 3}
$$

is the Pierce parameter, $I_{0}$ is the unperturbed beam current, $\mu$ is the parameter of inertial bunching, $K$ is the waveelectron coupling parameter proportional to the amplitude of the electron transverse oscillations, $\delta=\left(\bar{\omega}-\omega_{0}\right) / C \omega_{0}$ is the normalized mismatch of the Bragg frequency $\bar{\omega}=$ $\bar{h} c$ from the cutoff frequency, $N_{A, B}$ are the corresponding wave norms, and $\alpha$ is the wave coupling coefficient at the Bragg grating. For the coupling of two TE modes it can be put in a form

$$
\alpha_{T E \rightarrow T E}=\frac{r_{1}}{2 r_{0}^{2}} \frac{-\nu_{A}^{2}+m_{A} m_{B}}{\sqrt{h L} \sqrt{\left(\nu_{A}^{2}-m_{A}^{2}\right)\left(\nu^{2}-m^{2}\right)}} .
$$

Initial and boundary conditions to Eqs. (7) and (8) are given by

$$
\begin{gathered}
\left.\hat{B}\right|_{\tau=0}=\hat{B}_{0}, \\
\left.\hat{A}\right|_{Z=0}=0,\left.\quad \theta\right|_{Z=0}=\theta_{0}[0,2 \pi),\left.\quad \frac{\partial \theta}{\partial Z}\right|_{Z=0}=\Delta,
\end{gathered}
$$

where $L=l C \bar{h}$ is the normalized length of the resonator 
and $\Delta=\frac{\omega_{0}-h_{0} v_{\|}-\Omega}{\omega_{0} C}$ is the mismatch from synchronism between the electrons and the propagating wave at the carrier frequency.

Note that we supposed the characteristic time $Q / \omega$ of the changing of the trapped mode amplitude $B$ to be substantially greater than the electrons transit time $l / v_{\|}$and forward wave propagation time $l / v_{g r}$. In this case it is possible to assume that the amplitude of the trapped mode is constant during intervals $l / v_{\|}$and $l / v_{g r}$ and neglect time derivatives in the equations of electron motion (8) as well as the forward wave excitation equation (7a).

The electron efficiency of the oscillator is determined by the relations

$$
\begin{aligned}
& \eta=\frac{C}{\mu\left(1-\gamma_{0}^{-1}\right)} \hat{\eta}, \\
& \hat{\eta}=\left.\frac{1}{2 \pi} \int_{0}^{2 \pi}\left(\frac{\partial \theta}{\partial Z}-\Delta\right)\right|_{Z=L} d \theta_{0} .
\end{aligned}
$$

From Eqs. (7) and (8) one can obtain the energy conservation law

$$
\frac{\partial W}{\partial \tau}+P_{A}+P_{B}=4 \hat{\eta} .
$$

Here $W=|\hat{B}|^{2}$ is the normalized electromagnetic energy stored in the resonator, $P_{A}=|A(L)|^{2}$ represents radiation losses associated with the propagating wave, and the term $P_{B}=|\hat{B}|^{2} / Q$ represents losses associated with the trapped wave. It should be noted that the $Q$ factor of the trapped mode includes both diffraction and Ohmic losses.

\section{SELF-EXCITATION CONDITIONS AND ESTABLISHMENT OF THE STEADY-STATE OSCILLATION REGIME}

Obviously, when the coupling between modes is absent ( $\alpha=0)$ Eqs. (7) and (8) describe the convective mechanism of the electron beam-wave instability, and selfexcitation of the oscillations is impossible. Thus, the selfexcitation of the system is provided exceptionally by the coupling of the propagating wave with the cutoff trapped mode.

To get the self-excitation conditions we will approximate for simplicity the longitudinal structures of trapped modes as $f_{n}(Z)=\sin (\pi n Z / L)$, where $n=1,2,3 \ldots$ is longitudinal mode index. The amplitude of electron rf current under the assumption that the shifts of electron phases are small: $\theta=\theta_{0}+\vartheta\left(Z, \theta_{0}\right),|\vartheta| \ll 1$ can be reduced to the form

$$
\begin{aligned}
J & =\frac{1}{\pi} \int_{0}^{2 \pi} e^{-i \theta(Z)} d \theta_{0} \approx \frac{1}{\pi} \int_{0}^{2 \pi} e^{-i \theta_{0}}\left[1-i \vartheta\left(Z, \theta_{0}\right)\right] d \theta_{0} \\
& =\frac{-i}{\pi} \int_{0}^{2 \pi} e^{-i \theta_{0}} \vartheta d \theta_{0} .
\end{aligned}
$$

Correspondingly, using the linearized electron motion equations for the amplitude of electron current we get

$$
\left(\frac{\partial}{\partial Z}+i \Delta\right)^{2} J=-i \hat{A}
$$

with boundary conditions

$$
\left.J\right|_{Z=0}=0,\left.\quad \frac{\partial J}{\partial Z}\right|_{Z=0}=0 .
$$

In the case of precise electron-wave synchronism $\Delta=0$ the solution of linear equations (7a) and (10) with boundary conditions (11) can be presented in the form

$$
\begin{gathered}
J=\frac{\alpha \hat{B}}{2}\left(\frac{e^{i \delta+Z}}{(\delta+n \pi / L)^{3}-1}-\frac{e^{i \delta-Z}}{(\delta-n \pi / L)^{3}-1}\right)-\frac{\alpha \hat{B}}{6} \\
\times \sum_{m=1}^{3} e^{i \Gamma_{m} Z}\left(\frac{\Gamma_{m}}{\delta+n \pi / L-\Gamma_{m}}-\frac{\Gamma_{m}}{\delta-n \pi / L-\Gamma_{m}}\right), \\
A=i \frac{\partial^{2} J}{\partial Z^{2}} .
\end{gathered}
$$

Here $\Gamma_{m}$ are the roots of a well-known dispersion equation $\Gamma^{3}=1$ describing the normal waves in FEL with a forward propagating electromagnetic wave;

$$
\Gamma_{1}=1, \quad \Gamma_{2,3}=-\frac{1}{2} \pm \frac{\sqrt{3}}{2} i .
$$

Under the assumption that the length of the interaction space is rather large ( $L \gg 1$ ) the main contribution to the excitation factor is related with exponentially growing normal wave possessing the longitudinal wave number $\Gamma_{3}=-\frac{1}{2}+(\sqrt{3} / c) i$. Substituting this term into the righthand side of Eq. (7b) we obtain after integration over $Z$ the equation for amplitude of trapped mode $\hat{B}_{n}$ :

$$
\begin{aligned}
\frac{\partial \hat{B}_{n}}{\partial \tau}+\frac{1}{2 Q} \hat{B}_{n}= & \frac{\alpha^{2} \hat{B}_{n}}{3} e^{(\sqrt{3} L / 2)} \varphi_{n}(\delta, L) ; \\
\varphi_{n}(\delta, L)= & (-1)^{n}\left(\frac{1}{\delta+n \pi / L-\Gamma_{3}}-\frac{1}{\delta-n \pi / L-\Gamma_{3}}\right)^{2} \\
& \times e^{-(i L / 2)-i \delta L} .
\end{aligned}
$$

Therefore the self-excitation condition of the $n$th longitudinal mode can be presented in a form

$$
\frac{1}{2 Q}<\frac{\alpha^{2}}{3} e^{(\sqrt{3} L / 2)} \operatorname{Re} \varphi_{n}(\delta, L) .
$$

The function $\operatorname{Re} \varphi_{n}(\delta, L)$ for several longitudinal modes is plotted in Fig. 3. Self-excitation of the FEM is possible if the value of $\operatorname{Re} \varphi_{n}(\delta, L)$ is positive and exceeds factor $1 / 2 Q$. For sufficiently large $Q$ (or sufficiently small $\alpha$ ) the self-excitation region almost coincides with the region where the function $\operatorname{Re} \varphi_{n}(\delta, L)>0$. Note that the optimal starting length of the lowest mode $n=1$ is $L_{\mathrm{opt}}=2.5$ corresponding to $\delta_{\text {opt }} \approx 3$. 


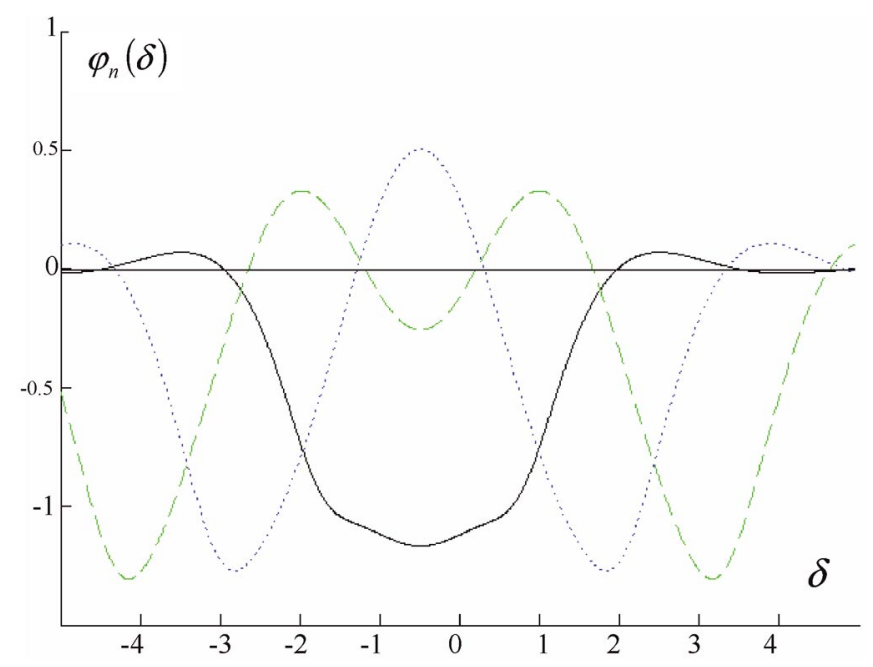

FIG. 3. (Color) Function $\varphi_{n}(\delta)$ for the first three modes at $L=$ 2.5. The black line corresponds to $n=1$, the blue line to $n=2$, and the green line to $n=3$.

We should emphasize that the excitation regions over mismatch $\delta$ for the modes with different longitudinal indices $n$ practically do not overlap (see Fig. 3). Therefore at fixed values $\delta$ and $L$ only a single longitudinal mode can be excited. It means that proposed FEM scheme possesses selectivity over longitudinal mode indices.

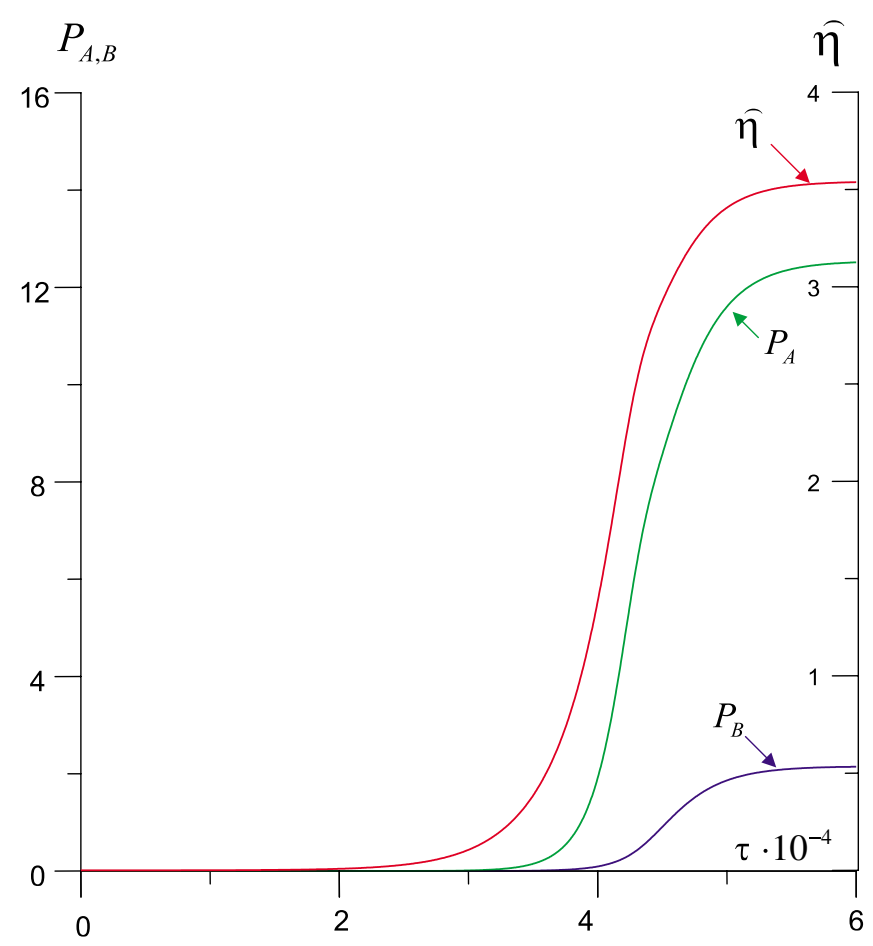

FIG. 4. (Color) Time dependences of the normalized electron efficiency $\hat{\eta}$ (red curve), radiation losses associated with propagating wave $P_{A}$ (green curve), and trapped wave $P_{B}$ (blue curve) for $L_{\mathrm{opt}}=2.5, \delta \approx 3, \Delta \approx-0.6$.

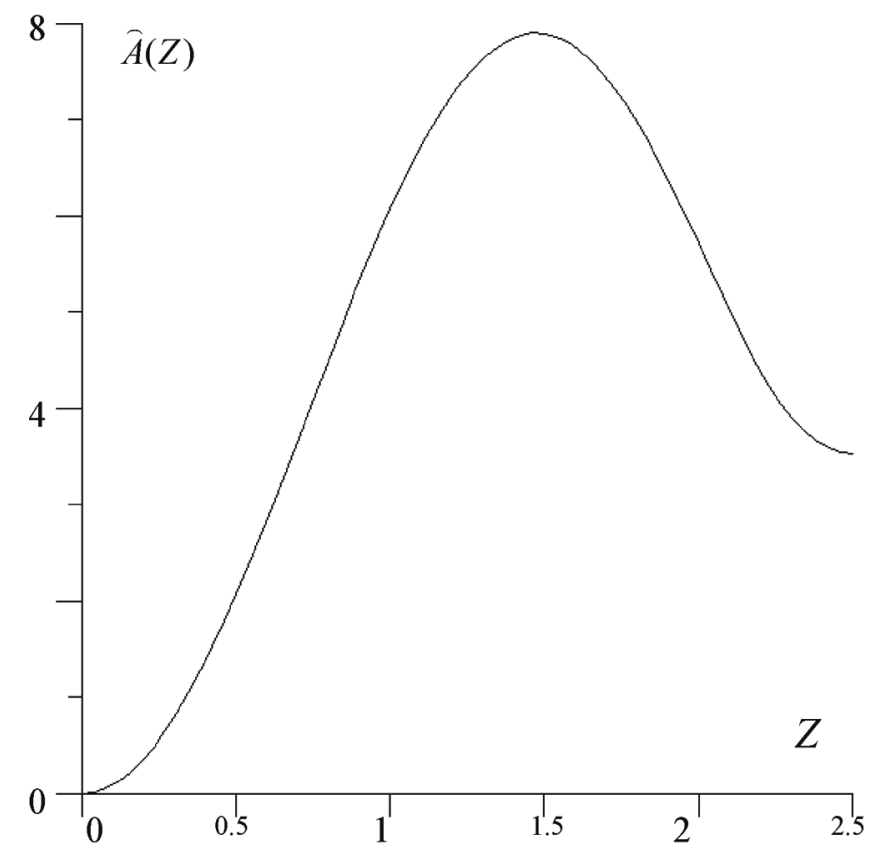

FIG. 5. Longitudinal structure of the propagating wave $A(Z)$ in a steady-state regime for $L_{\text {opt }}=2.5, \delta \approx 3, \Delta \approx-0.6$.

Results of numerical simulation of the nonlinear stage of interaction based on Eqs. (7) and (8) are shown in Figs. 4 and 5 for the first longitudinal mode $n=1$. Establishment of a stationary regime of generation can be seen in Fig. 4. In this regime the spatial structure of the propagating mode is plotted in Fig. 5. Normalized efficiency is rather high $\hat{\eta} \sim 3$. It is important to note that in optimal conditions the main part of the energy extracted from electron beam transforms in the radiation of the propagating mode $A$ but not dissipated with the trapped mode $B$. Variation of electron mismatch $\Delta$ can be used for further optimization of the electron efficiency.

\section{CONCLUSION}

Using the above analysis let us estimate a possibility of creation of a $4 \mathrm{~mm}$ FEM on the basis of the accelerator LIU-3000 (JINR, Dubna) (1 MeV/200 A/200 ns). Taking waveguide radius $a \approx 0.62 \mathrm{~cm}$, the period of undulator $d_{u} \approx 3.8 \mathrm{~cm}$ and the period of helical corrugation $\bar{m}=$ $4, d \approx 0.41 \mathrm{~cm}$ we find that the conditions of a Bragg resonance (4) are fulfilled for the pair of modes $T E_{11} \rightarrow$ $T M_{32}\left(\nu_{A}=1.82, \nu_{B}=9.76\right)$. In the case of Pierce parameter $C \approx 10^{-2}$ the length of interaction space $l=$ $15.3 \mathrm{~cm}$ and corrugation depth $a_{1} \approx 0.01 \mathrm{~cm}$ correspond to the normalized length $L=2.5$ and the coupling parameter $\alpha=0.1$. The relative mismatch of the Bragg frequency from the cutoff frequency $\left(\bar{\omega}-\omega_{0}\right) / \omega_{0} \approx 0.03$ provides normalized mismatch $\delta \approx 3$. The establishment of steadystate regime for above normalized parameters just shown in Fig. 4. The normalized efficiency $\hat{\eta}=3$ corresponds to 
the total efficiency $\eta \approx 15 \%$. The transient time occupies about $100 \mathrm{~ns}$.

As follows from the above consideration extremely shallow corrugation $\left(a_{1} / a<0.02\right)$ is sufficient for FEM self-excitation if the trapped cutoff mode is used in the feedback loop. Thus the using of a cutoff mode makes it possible to decrease effective coupling parameter sufficiently enough for the self-excitation of the oscillator in comparison with traditional Bragg FEM scheme, where feedback wave possesses rather high group velocity [6]. Correspondingly, in oversized microwave system where Bragg conditions are satisfied for a large number of pairs of waves with different transverse indices it is possible to provide selective excitation of a single pair consisting of a cutoff mode and operating propagating mode which is amplified by the electron beam. The above method of mode control will be tested in JINR-IAP FEM $[7,8]$ at $\mathrm{Ka}$ band and then used as a method of increasing the operating frequency to $W$ band for a fixed transverse size.

\section{ACKNOWLEDGMENTS}

This work is partially supported by INTAS and the Russian Foundation for Basic Research (Grants No. 0302-16530 and No. 04-02-17118).
[1] A. L. Goldenberg, G.N. Nusinovich, and A.B. Pavelyev, in Gyrotrons (IAP RAS, Gorky, Russia, 1980), pp. 91-97.

[2] G.G. Denisov, V.L. Bratman, A.D.R. Phelps, S.V. Samsonov, IEEE Trans. Plasma Sci. 26, 508 (1998).

[3] A. V. Savilov, V.L. Bratman, A.D. R. Phelps, and S. V. Samsonov, Phys. Rev. E 62, 4207 (2000).

[4] A. V. Savilov, N. Yu. Peskov, and S.V. Samsonov, Nucl. Instrum. Methods Phys. Res., Sect. A 445, 284 (2000).

[5] N. S. Ginzburg, A M. Malkin, N.Yu. Peskov, and A. S. Sergeev, in Book of Abstracts of the 15th International Conference on High-Power Particle Beams, St. Petersburg, Russia, 2004 (D. V. Efremov Scientific Research Institute of Electrophysical Apparatus, St. Petersburg, 2004), p. 164.

[6] N. S. Ginzburg, V. L. Bratman, G. G. Denisov, and M.I. Petelin, IEEE J. Quantum Electron. 19, 282 (1983).

[7] N. S. Ginzburg, A. A. Kaminsky, A. K. Kaminsky, N. Yu. Peskov, S. N. Sedykh, A.P. Sergeev, and A. S. Sergeev, Phys. Rev. Lett. 84, 3574 (2000).

[8] A. K. Kaminsky, A. V. Elzhov, N.S. Ginzburg, S. V. Kuzikov, E. A. Perelstein, N. Yu. Peskov, M. I. Petelin, S.N. Sedykh, A.P. Sergeev, A.S. Sergeev, I. V. Syratchev, and N.I. Zaitsev, Nucl. Instrum. Methods Phys. Res., Sect. A 528, 78 (2004). 